makes the interpretation of any resulting phenomenology extremely difficult.

It is also clear from the original publication by Singleton et al that no specific assessment for PTSD was carried out, although validated and reliable instruments for this exist (e.g. the Clinical Assessment for PTSD, Blake et al (1995) or the Posttraumatic Stress Symptoms Interview (PSSI) or Posttraumatic Stress Symptoms Self-Report (PSS-SR), Foa et al (1993)). The authors did a partial screen for a few recognised PTSD symptoms, such as reexperiencing and avoidance, but there was no systematic assessment of the condition that would have allowed them to diagnose the full disorder. It should be recognised that PTSD is a major psychiatric disorder that constitutes a serious burden for the individual and for society (Kessler, 2000). A diagnosis of PTSD has implications in terms of assessing the individual's risk and in terms of treatment recommendations. It is important that the term post-traumatic stress should not be confused or conflated with the term 'post-traumatic stress disorder'. The description of post-traumatic stress made by Coid et al cannot be evaluated without deconstructing more precisely what this means. As there are now well-recognised instruments to assess PTSD and lifetime experience of traumatic events in a range of settings, without these being used then terms such as post-traumatic stress should be avoided.

American Psychiatric Association (1994) Diagnostic and Statistical Manual of Mental Disorders (4th edn) (DSM-IV). Washington, DC: APA.

Blake, D. D., Weathers, F., Nagy, L., et al (1995) The development of a clinician administered PTSD scale. Journal of Traumatic Stress, 8, 75-90.

Coid, J., Petruckevitch, A., Bebbington, P., et al (2002a) Ethnic differences in prisoners. I: Criminality and psychiatric morbidity. British Journal of Psychiatry I8I, 473-480.

$\ldots, \ldots, \ldots$, et al (2002b) Ethnic differences in prisoners. 2: Risk factors and psychiatric service use. British Journal of Psychiatry, 18I, 48I-487.

Foa, E. B., Riggs, D. S., Dancu, C. V., et al (1993)

Reliability and validity of a brief instrument for assessing posttraumatic stress disorder. Journal of Traumatic Stress, 6, 459-473.

Kessler, R. G. (2000) Posttraumatic stress disorder: the burden to the individual and to society. Journal of Clinical Psychiatry, 61 (suppl. 5), 4-12.

Singleton, N., Meltzer, H. \& Gatward, R. (1998) Psychiatric Morbidity Among Prisoners in England and Wales. London: Stationery Office.

G. Mezey Forensic and Personality Disorder Group, Department of Psychiatry, St George's
Hospital Medical School, Jenner Wing, Cranmer Terrace, London SWI7 ORE, UK

\section{Specialist care for prisoners?}

In his recent editorial on mental health in prisons Dr Reed (2003) urges, understandably and in most cases correctly, that the quicker that patients with psychosis are transferred to specialist psychiatric care, the better.

However, there are prisoners with schizophrenia, willing to take medication, who survive reasonably comfortably in the prison milieu. Their great fear is that they will be transferred to a special psychiatric hospital; 'nutted off' in prisonspeak. They have reason to fear a transfer, for it effectively exchanges a finite sentence for an indefinite one. In the case of those serving a life sentence, it means their fate is in the hands of a mental health review tribunal rather than the Parole Board, the latter, they believe, being less cautious in recommending discharge. As an ex-member of both organisations, I would agree with them.

So, while prison is obviously bad for people with mental illness, hospital is sometimes worse.

Reed, J. (2003) Mental health care in prisons. British Journal of Psychiatry, 182, 287-288.

A. Gibson correspondence c/o the British Journal of Psychiatry, 17 Belgrave Square, London SWIX 8PG, UK

\section{Consent and treatment in prisons}

I read the article by Earthrowl et al (2003) with interest. The issue of providing treatment to prisoners, who are frequently incapable of consenting, will not be unfamiliar to psychiatrists providing mental health care in these establishments. Although the authors correctly state that there is no legislative framework for providing treatment for mental disorders in prisons, this may be slightly disingenuous. The current legislative framework that provides for the treatment of mental disorders, namely the Mental Health Act 1983, is clear that prison health-care wings are not hospitals. It follows that any treatment that is administered forcibly must be consistent with common law. Separate legislation is therefore unnecessary.

They also appear to have overlooked recent guidance on this matter. The Department of Health (2002) in collaboration with the Prison Service has set out, in detail, good practice guidelines for providing care to both competent and incapacitated adult prisoners. These outline circumstances in which prisoners who lack capacity can receive treatment. We have found this very helpful in developing protocols for treatment in the prisons we visit.

The development of policies and protocols will assist in establishing who, when and in which circumstances incapacitated prisoners may be treated and allow us to be more confident when making these difficult decisions.

Department of Health (2002) Seeking Consent: Working with People in Prison. London: Department of Health

Earthrowl, M., O'Grady, J. \& Birmingham, L. (2003) Providing treatment to prisoners with mental disorders: development of a policy. Selective literature review and expert consultation exercise. British Journal of Psychiatry, 182, 299-302.

I. Qurashi Gulid Lodge,Whittingham Lane, Preston PR3 2AZ, UK

Authors' reply: We fail to understand $\mathrm{Dr}$ Qurashi's comment that 'separate legislation is therefore unnecessary'. Our paper sets out a policy for providing treatment to people with mental disorder based on common law (Earthrowl et al, 2003). We are not proposing separate legislation.

Dr Qurashi also mentions that we appear to have overlooked recent guidance from the Department of Health (2002). The Department of Health guidelines were produced in July 2002, after our paper was accepted for publication.

These guidelines provide guidance on establishing capacity but, in our opinion, they do not tackle the practical issues relating to the management of prisoners with mental disorder in any great detail, they do not deal with the ethical issues surrounding the provision of an equivalent service in prisons adequately and detailed guidance on making a concerted effort to obtain treatment under the Mental Health Act in hospital before proceeding with treatment under common law is lacking. In our view, these are serious omissions.

\section{Declaration of interest}

J.O. is a member of the Department of Health Prison Expert Group. 